\title{
Efecto cardioprotector del ejercicio aeróbico en personas con riesgo de hipertensión arterial ${ }^{1}$
}

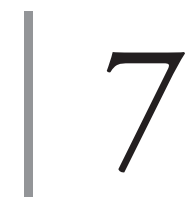

\author{
https://doi.org/10.21830/9789585284814.07
}

Daniel Eduardo Arévalo Contreras', Diogo Rodrigues Bezerra ${ }^{3}$

\section{Resumen}

La presente investigación busca evidenciar el beneficio del ejercicio aeróbico sobre la salud cardiovascular de personas con riesgo de hipertensión arterial (HTA). Objetivo: el objetivo principal consistió en determinar el efecto cardioprotector sobre la presión arterial (PA) en reposo, por medio de ejercicios de resistencia aeróbica mediante el método continuo a dos intensidades para la correcta planificación del entrenamiento en personas con riesgo de HTA, evidenciado a través de la disminución en la PA por debajo de los niveles de reposo que ocurre luego del ejercicio físico, denominándose hipotensión postejercicio (HPE). Metodología: los participantes fueron 8 sujetos sedentarios y en estado de sobrepeso u obesidad determinados como factores de riesgo de HTA, quienes participaron en 2 sesiones de ejercicio en bicicletas estáticas con intensidades del 50 y $70 \%$ de la frecuencia cardiaca de reserva y una sesión control. Cada sesión constó de 10 minutos en reposo, un volumen de 30 minutos de ejercicio y posteriormente mediciones de pa cada 10 minutos durante 1 hora mediante métodos no invasivos. Resultados: se evidenciaron diferencias estadísticamente significativas en la presión arterial sistólica (PAS), tanto al 50\% como al $70 \%$ en comparación con la presión arterial diastólica (PAD), sin embargo, la mayor disminución se obtuvo en la intensidad del $70 \%$ de ejercicio. Conclusión: se comprueba la incidencia del ejercicio aeróbico sobre la PA independientemente de la intensidad mediante la HPE, no obstante, se determinó únicamente para la PAS.

Palabras clave: ejercicio aeróbico; hipertensión arterial; hipotensión post-ejercicio; presión arterial.

1 Capìtulo original, desarrollo de una tesis de pregado del programa Licenciatura en Ciencias del Deporte de la Universidad Manuela Beltrán.

2 Licenciado en Ciencias del Deporte de la Universidad Manuela Beltrán. Orcid: https://orcid. org/0000-0002-1740-076X

3 Magister en Actividad Física y Salud, Docente y Coordinador de Investigaciones en el Programa de Licenciatura en Ciencias del Deporte de la Universidad Manuela Beltrán. Contacto: diogo.rodrigues@ docentes.umb.edu.co. Orcid: https://orcid.org/0000-0002-3001-7597 


\section{Introducción}

El desarrollo de la sociedad ha modificado diferentes aspectos en las personas, lo que repercute en sus estilos de vida y las condiciones de salubridad; según Hernán et al., (2015, p. 162) "la inactividad física se ha incrementado de forma considerable en la sociedad actual pudiendo acarrear efectos perjudiciales para la salud". Algunos de estos problemas son cardiovasculares, causados principalmente por el sedentarismo, lo que puede conllevar a una obesidad en el individuo, relacionándose esta última con una mayor prevał lencia de hipertensión arterial (HTA) (González et al., 2015, p. 275), la cual es, según Briones (2016, p. 36), una enfermedad caracterizada por la elevación de la presión arterial sistólica (PAS) mayor a 140 milímetros de mercurio $(\mathrm{mmHg})$ y/o presión arterial diastólica (PAD) mayor a $90 \mathrm{mmHg}$.

Según el American College of Sports Medicine (ACSM) (2004), la "presión arterial (PA) en reposo, historia familiar de HTA, índice de masa corporal y la actividad física y la aptitud son generalmente aceptados indicadores de futura HTA” (p. 535), siendo necesario determinar, por medio de estos factores, el grado de riesgo de la persona a desarrollar esta enfermedad, ya que de acuerdo con el Ministerio de Salud y Protección Social (Minsalud) (2014, p. 144) la HTA causa 9,4 millones de muertes anualmente en el mundo.

El ejercicio físico aeróbico funciona como una opción de gran relevancia en la prevención y el tratamiento no farmacológico de la HTA (Simão et al., 2007 , p. 117) produciendo diferentes adaptaciones en el organismo; una de ellas se puede evidenciar por medio de la hipotensión post-ejercicio (HPE), que se define como "la disminución en la presión arterial por debajo de los niveles de reposo que ocurre luego del ejercicio físico" (Veloso et al., 2009, p. 496), razón por la cual es recomendado para la prevención de enfermedades cardiovasculares (Cornelissen, Verheyden, Aubert \& Fagard, 2010, p. 175).

En cuanto a las respuestas hipotensoras posterior al ejercicio, de acuerdo con Pescatello et al., (2004, p. 540) no se han evidenciado diferencias significativas entre personas con diferentes etnias, por lo tanto, este efecto cumple con la generalización de los resultados independientemente de la procedencia de cada persona. Sin embargo, la raza del sujeto es un factor determinante, ya que 
se ha evidenciado, según Mora (2010), que "la población afroamericana tiene unos niveles de presión arterial superiores a la población caucásica probablemente debido a un mayor tono del sistema simpático” (p. 135). Por otro lado, en lo referente a la relación de la HPE entre hombres y mujeres no se han determinado diferencias significativas más allá de la recuperación más acelerada de los hombres con respecto a las mujeres (Chiacchio, Ricart \& Suau, 2010).

Con base en diversos estudios realizados para analizar este fenómeno, el presente artículo ha estipulado como objetivo analizar los efectos hipotensivo posterior al esfuerzo físico en diferentes momentos.

\section{Respuestas vasculares}

Debido a que un condicionante de la PA es el grado de resistencia vascular periférica, esta es modificada por múltiples procesos en el momento de la ejecución del ejercicio y luego del mismo, dentro de estas se hace referencia a disminuciones en la actividad nerviosa simpática periférica, la disminución de catecolaminas circundantes y la disipación de calor, entre otras (Simão et al., 2007, p. 120).

De otro lado, según Pescatello et al. (2004, p. 543), durante la realización de ejercicio físico se ha evidenciado el aumento de sustancias vasodilatadoras, como lo es el óxido nítrico, en sujetos sanos, por tanto, después del ejercicio disminuyen la resistencia vascular total y posteriormente la PA de los individuos.

\section{Sistema renina angiotensina}

Por otra parte, Cornelissen y Fagard (2005) mencionan que la práctica de ejercicio de resistencia aeróbica realiza HPE al reducir la resistencia vascular, por posibles implicaciones del Sistema renina-Angiotensina, ya que, al ser un potente vasoconstrictor, "la renina es liberada de los rińones durante periodos de baja presión de perfusión" (MacDonald, J. 2002, p. 231) por lo cual, debido a las demandas en el ejercicio, aumenta la vasoconstricción gracias a este proceso y posterior al ejercicio disminuye para la regulación de la PA, pudiendo así ser un precursor de hipotensión posterior al esfuerzo físico. No 
obstante, Pescatello et al., (2004, p. 543) mencionan que la realización de ejercicio no disminuye significativamente los niveles de renina y angiotensina, evidenciándose que este sistema no favorece principalmente la disminución de la PA finalizado el ejercicio.

\section{Catecolaminas}

Durante la suspensión del ejercicio dinámico, los procesos influenciados por las catecolaminas disminuyen (vasoconstricción, aumento de la frecuencia cardiaca (FC) y aumento de la contractilidad miocárdica), pero su influencia en la HPE es mínima (MacDonald, J. 2002, p. 231). Por otro lado, Del Valle et al., (2015) sustentan que otras adaptaciones vasculares realizadas en el ejercicio para la disminución de la pa son debidas a "un descenso de la estimulación de los receptores alfa-adrenérgicos" (p. 295). En todo caso, "en general se acepta que los mecanismos subyacentes a la disminución sostenida de la PA de los hipertensos después de entrenamiento son una disminución de la FC en reposo y una disminución de catecolaminas circulantes" (Tipton, 1984 citado en MacDonald, 2002, p. 226).

\section{Adaptaciones estructurales}

Con base en las adaptaciones ocasionadas por el ejercicio físico a nivel cardiovascular, que favorecen la aparición de respuestas hipotensoras, se han evidenciado, según Pescatello et al. (2004), remodelaciones vasculares —en cuanto longitud y diámetro- de las arterias y venas existentes (p. 544), favoreciendo así la disminución de la resistencia vascular periférica.

\section{Resistencia a la insulina e hiperinsulinemia}

Según Cornelissen y Fagard (2005), el entrenamiento de resistencia aeróbica repercute favorablemente en la sensibilidad a la insulina (p. 672), razón por la cual, debido a que esta hormona está relacionada con la activación del sistema nervioso simpático y con la HTA, al disminuir la resistencia a la insulina, paralelamente disminuye el flujo simpático y, por ende, se reduce la PA (Pescatello et al., 2004, p. 543) 


\section{Termorregulación}

Un mecanismo para la liberación de calor en el organismo es la vasodilatación cutánea, ya que esta junto con la vasoconstricción permite la homeostasis de la temperatura corporal (Estañol et al., 2016, p. 404). "Dado que la temperatura del cuerpo aumenta con el ejercicio, es posible que una redistribución de la sangre a la periferia pueda ser responsable de la HPE”, según MacDonald (2002 p. 229), sin embargo, el mismo autor menciona que "es poco probable que la vasodilatación cutánea sea el principal mecanismo responsable de la HPE”.

Ahora bien, de acuerdo con Gomes y Doederlein (2010), quienes mencionan que "no hay pruebas sobre el exacto mecanismo que está involucrado en la HPE" (p. 107), se observa un efecto multisistémico del ejercicio sobre el organismo para producir dicha respuesta.

De acuerdo con Gamboa y Solera (2014, p. 102), se han llevado a cabo estudios donde se investiga el efecto agudo del ejercicio físico sobre la PA, sin embargo, aún existen dudas en cuanto a la indicación de este, donde ocupa un rol importante la intensidad a la cual se ejecute, por lo cual es necesario diferenciar este efecto en diferentes poblaciones, ya que las condiciones de cada individuo o grupo poblacional difieren entre sí. Por lo tanto, es necesario determinar cuál es la intensidad más adecuada en ejercicio aeróbico para obtener el mejor efecto cardioprotector en personas con riesgo de hipertensión arterial.

En consecuencia, es indispensable para el profesional del ejercicio físico poseer conocimientos adecuados y suficientes para la intervención a personas con factores de riesgo de HTA, por medio de la regulación de las cargas y sus implicaciones desde el punto de vista del diseño y desarrollo del programa de entrenamiento (Márquez \& Garatachea, 2009, p. 306), omitiendo así la improvisación en la aplicación de los ejercicios y previniendo esta enfermedad, contribuyendo paralelamente en la calidad de vida de los individuos.

Por todo lo anterior, el objetivo de este estudio es determinar el efecto cardioprotector sobre la presión arterial en reposo, por medio de ejercicios de resistencia aeróbica mediante el método continuo a dos intensidades, para la correcta planificación del entrenamiento en personas con riesgo de hipertensión 
arterial. Así mismo, se buscan los siguientes objetivos específicos: a) comparar el efecto agudo en la presión arterial posterior a la realización de ejercicio aeróbico a diferentes intensidades; b) analizar la variabilidad del efecto agudo post-ejercicio sobre la presión arterial según el tiempo de toma de esta y c) comparar la magnitud del efecto hipotensivo post-ejercicio entre hombres y mujeres.

\section{Metodología}

La presente investigación es de diseño experimental, tipo de estudio longitudinal con enfoque cuantitativo de alcance descriptivo; la muestra del estudio realizado fue no probabilística por conveniencia. Fue aprobado por el comité de ética de la Universidad Manuela Beltrán y el procedimiento se basó en los aspectos éticos de la declaración Helsinki.

Con respecto al tipo de investigación, se considera cuantitativo bajo un diseño cuasi-experimental, en donde la identificación de la población se realizó por medio de muestras no probabilísticas, se seleccionaron 16 sujetos con una edad promedio de $31,1 \pm 8,4$ años, un peso de 80,1 $\pm 11,4 \mathrm{~kg}$, estatura 1,66 \pm 0,05 metros y un índice de masa corporal (IMC) 28,9 $\pm 3,1$.

Para poder participar en el estudio se determinaron los siguientes criterios de inclusión: a) ser normotenso, con el objetivo de evitar la influencia de algún medicamento antihipertensivo en el resultado; b) tener un IMC mayor a $25 \mathrm{Kg} /$ $\mathrm{m}^{2}$; c) ser mayor de edad; d) presentar una PAS en reposo $\leq 140 \mathrm{mmHg}$ y PAD $\leq$ $90 \mathrm{mmHg}$; e) ser sedentario, es decir, no realizar ejercicio mayor a 3 veces por semana y con una duración de 30 minutos por sesión.

Por otra parte, los criterios de exclusión fueron: a) tener patologías cardiacas o presentar enfermedades metabólicas (hiperlipidemia, diabetes mellitus); b) ser fumadores activos, con el objetivo de evitar un posible efecto de la nicotina sobre los resultados; c) individuos bajo tratamiento farmacológico de acción inotrópica y/o cronotrópica, para no tener alteración de resultados en cuanto a la PA y la FC, respectivamente; d) haber consumido bebidas con cafeína previo a la intervención, ya que se ha observado que esta provoca elevación temporal de la PA (Del Valle et al., 2015, p. 295); e) tomar bebidas energizantes antes de la prueba. 


\section{Procedimientos}

El ejercicio aeróbico se realizó mediante la utilización de bicicletas estáticas, realizándose el control de intensidad del ejercicio por medio de monitores de FC marca Polar FT1, basándonos en los cálculos obtenidos por la fórmula de Frecuencia Cardiaca Máxima (FCM) de Tanaka, Monahan y Seals (2001) $\mathrm{FCM}=\{208,75-0,73 *$ edad $\}$ ya que es una de las más exactas para diferentes tipos de población (Marins \& Delgado, 2007, p. 118). Paralelamente, se calculó la FC que debe oscilar durante el ejercicio en determinada intensidad, por medio de la fórmula de Karvonen: $\mathrm{FCE}=(\mathrm{FC}$ máx $-\mathrm{FC}$ reposo $)(\%$ intensidad del ejercicio) + FC reposo.

La medición de la PA se realizó por medio de un método no invasivo, permitiendo obtener una valoración cuantitativa de la PA post-ejercicio, mediante un tensiómetro digital Marca OWNERs Model BP-1319, ya que, de acuerdo con lo estipulado por Chiacchio, Ricart y Suau (2010), "la determinación de la TA mediante aparatos automáticos, con la técnica correcta brindan muchas ventajas sobre la técnica manual" (p. 192).

En virtud de lo anterior, se estableció la medición sugerida por Simão et al., (2007, p. 119) quienes mencionan la posición del manguito del tensiómetro donde el borde inferior debe estar aproximadamente a $2 \mathrm{~cm}$ por encima de la fosa antecubital, siendo colocado en el brazo izquierdo; por otra parte, la postura de los individuos fue en posición sentada, ya que cuando se realiza una variación de la posición "se producen ajustes cardiovasculares a consecuencia de la acción de la gravedad que inciden en los valores de presión" (Calderón, 2009, p. 79), como resultado se obtuvieron registros de: PAS en mmHg, PAD en $\mathrm{mmHg}$ y FC expresada en pulsaciones por minuto (ppm).

Cada individuo asistió a 3 sesiones individuales en distintos días con lapsos de 72 horas entre cada una; con anticipación se solicitó a los participantes no realizar ejercicio físico previo a la prueba, ni consumir cafeína para la valoración de la PA.

Se midió la PA en reposo en posición sentado y con 10 minutos de inactividad física, posteriormente se ejecutó el ejercicio físico a una intensidad por día de manera progresiva para valorar la continuidad de cada participante y su respuesta cardiovascular en el siguiente orden: 50 y $70 \%$ de intensidad de 
acuerdo con su FC máx.; por último, se realizó la prueba de control en la que no se llevó a cabo ningún esfuerzo físico. El volumen de las diferentes pruebas fue de 30 minutos por sesión, con intensidad constante bajo el método continuo, lo que hace necesario tener monitorización de FC constante para su control.

Cuando finalizó el ejercicio se continuó con el protocolo realizado por Gamboa y Solera (2014, pp. 102-103), que consistió en tomar la PA finalizando inmediatamente la prueba con el individuo sentado, luego se tomó cada 10 minutos hasta completar los 60 minutos, de igual manera las muestras fueron manejadas de manera anónima, asegurando la imposibilidad de inferir su identidad, para su estudio y potencial análisis ulterior, de esta forma se garantiza la confidencialidad de los datos entre otros.

Durante la prueba de ejercicio físico dentro del estudio, se confirmó el estado de salud del participante declarándose aparentemente sano, sin patologías cardiovasculares y/o enfermedades relacionadas que pudiesen tener complicaciones, no obstante, debido a la naturaleza del estudio y de acuerdo con la Ley 8430 de 1993, existen riesgos mínimos de exposición, siendo avalado el día 5 de junio de 2017 por el comité de ética de investigaciones de la Universidad Manuela Beltrán con acta de evaluación No 05-0605-2017.

\section{Resultados}

A partir de la estadística descriptiva se obtuvieron promedios y desviaciones estándar para la PAS y PAD según la condición del ejercicio realizada, como se evidencia en la Tabla 1.

Tabla 1. Estadística descriptiva de la PA según la condición y el momento de medición

\begin{tabular}{lcccccc}
\hline & \multicolumn{3}{c}{ TAS } & & \multicolumn{3}{c}{ TAD } \\
\hline Mediciones & Control & $\mathbf{5 0} \%$ & $\mathbf{7 0} \%$ & Control & $\mathbf{5 0} \%$ & $\mathbf{7 0 \%}$ \\
\hline FCR & $124,3 \pm 3,2$ & $125 \pm 3,1$ & $123,2 \pm 10,5$ & $81 \pm 4,6$ & $80,5 \pm 3,3$ & $81,2 \pm 9,5$ \\
FE & $125,3 \pm 1,9$ & $126,7 \pm 9,4$ & $130,7 \pm 11,4$ & $81 \pm 3,3$ & $78,2 \pm 10,1$ & $80,5 \pm 7,9$ \\
10 min & $125,9 \pm 4,4$ & $118 \pm 10,5$ & $116,2 \pm 9,7$ & $81,2 \pm 4,8$ & $74,5 \pm 8,7$ & $77 \pm 6,0$ \\
\hline
\end{tabular}




\begin{tabular}{lcccccc}
\hline & \multicolumn{3}{c}{ TAS } & \multicolumn{3}{c}{ TAD } \\
\hline Mediciones & Control & $\mathbf{5 0} \%$ & $\mathbf{7 0} \%$ & Control & $\mathbf{5 0} \%$ & $\mathbf{7 0} \%$ \\
\hline 20 min & $121,3 \pm 4,0$ & $118,3 \pm 8,1$ & $117,3 \pm 6,7$ & $79,8 \pm 6,8$ & $73,3 \pm 8,2$ & $75,3 \pm 8,7$ \\
30 min & $121,8 \pm 2,1$ & $117,8 \pm 11,3$ & $118,7 \pm 6,7$ & $80.2 \pm 4,5$ & $78,7 \pm 8,8$ & $77,7 \pm 7,1$ \\
40 min & $125 \pm 7,2$ & $118,2 \pm 9,5$ & $119,5 \pm 6,7$ & $81,2 \pm 2,7$ & $80 \pm 6,9$ & $74 \pm 7,9$ \\
50 min & $125 \pm 3,5$ & $119,2 \pm 8,0$ & $118 \pm 8,1$ & $81,7 \pm 3,1$ & $75,8 \pm 11,1$ & $77,5 \pm 5,7$ \\
60 min & $125 \pm 3,1$ & $120 \pm 7,0$ & $121 \pm 10,5$ & $80,5 \pm 3,3$ & $77,5 \pm 10,5$ & $80,2 \pm 10,5$ \\
\hline
\end{tabular}

FCR: Frecuencia cardiaca de reposo; FE: Finalizado el ejercicio; PAS: presión arterial sistólica; PAD: presión arterial diastólica.

Fuente: elaboración propia.

Asimismo, en la Figura 1 se evidencian las variaciones de la PAS, de acuerdo con las diferentes intensidades del ejercicio como del momento de su toma; a este respecto, se muestra una mayor disminución de la PAS a una intensidad del $70 \%$ en el minuto 10 posterior a la finalización del ejercicio, indicando un valor de 116,2 mmgH. Sin embargo, la disminución de la PAS con intensidad del $50 \%$ obtuvo una respuesta hipotensiva similar en el mismo momento, disminuyendo hasta alcanzar $118 \mathrm{mmHg}$, teniendo como diferencia $1,8 \mathrm{mmHg}$ en comparación con el $70 \%$. En los momentos posteriores a los 10 minutos finalizado el ejercicio, se observa una recuperación paulatina tanto de la PAS al 70\% como al 50\%, no obstante, dentro de los 60 minutos posteriores al ejercicio, la PAs no retomó sus valores iniciales en ninguna de las dos intensidades con respecto a la toma previa del esfuerzo.

Contrastando las dos intensidades del ejercicio a partir de la toma de control, se evidencia un descenso de la PAs de $7,9 \mathrm{mmHg}$ y $9,7 \mathrm{mmHg}$ al 50 $\%$ y $70 \%$, respectivamente, en el minuto 10 finalizado el ejercicio, siendo este, como se mencionó previamente, el mayor descenso que se obtuvo durante la recolección de datos.

Por otro lado, dentro del efecto post-ejercicio sobre la PAD se evidenciaron disminuciones de esta a partir del 50 y el $70 \%$ de intensidad del ejercicio, determinando así un mayor efecto hipotensivo con una diferencia de $6,5 \mathrm{mmHg}$ en el minuto 20 y 7,2 mmHg en el minuto 40, respectivamente para cada intensidad con respecto a la sesión control, como se evidencia en la Figura 2. 


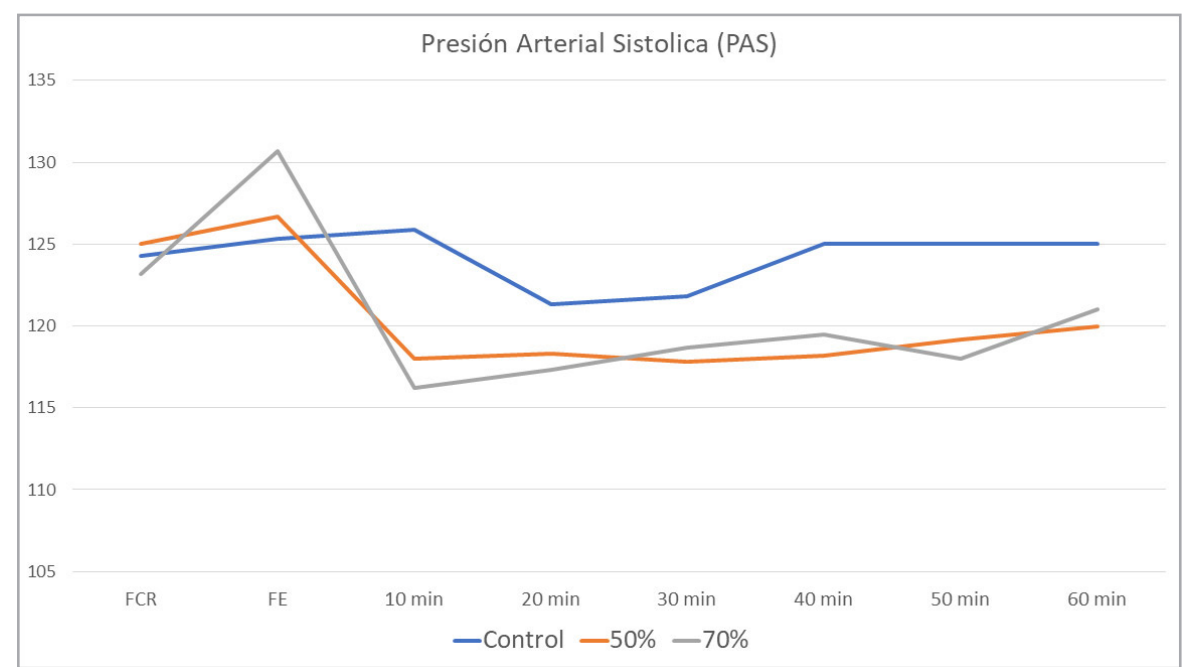

Figura 1. Variaciones de la presión arterial sistólica de acuerdo con las diferentes intensidades del ejercicio.

Fuente: elaboración propia.

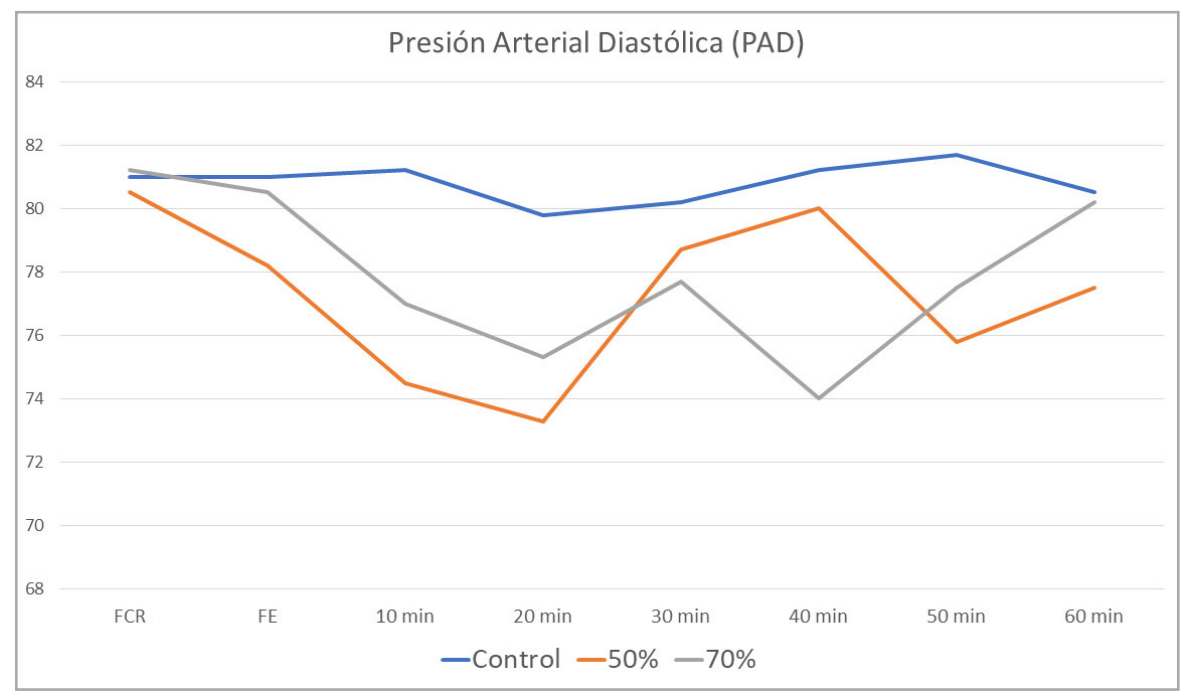

Figura 2. Variaciones de la presión arterial diastólica de acuerdo con las diferentes intensidades del ejercicio.

Fuente: elaboración propia.

Igualmente, en la recuperación de la PAD, no se evidencia una progresión uniforme para el recobro de sus valores iniciales. En la PAD sometida a un ejer- 
cicio con intensidad del $70 \%$ se observa una recuperación completa a los 60 minutos posteriores a la realización del ejercicio, en cambio, la recuperación a intensidad del 50\% FCR no se recuperó en su totalidad en el mismo momento de toma.

Asimismo, en el estado de reposo previo a cada sesión no se evidencian diferencias determinantes entre cada condición, lo que indica que los sujetos iniciaron las 3 sesiones con PA similares.

Por otra parte, dentro de la sesión control se evidencia la mayor diferencia entre hombres y mujeres dando como diferencia $9,8 \mathrm{mmHg}$ en el minuto 40, siendo la PAs más elevada en los hombres. Sin embargo, en lo que atañe a la comparación de la respuesta hipotensora de acuerdo con el género, no se evidencian diferencias relevantes con respecto a efecto hipotensivo ni a la recuperación de la $\mathrm{PA}$.

Del mismo modo, el análisis descriptivo de la respuesta de la PAD evidenció que dentro de la sesión control sin ejecución de esfuerzo físico no obtuvieron el mismo comportamiento; asimismo, en la sesión del 50\% se observa disminución de la PAD con respecto a los valores iniciales, pero no se evidencia una recuperación con las mismas características en cuanto a su progresión.

Para utilizar un diseño de experimentos fue necesario comprobar los supuestos, determinándose a través de pruebas de normalidad y análisis de varianzas.

En cuanto a las pruebas de normalidad de los datos se realizó el test de Kolmogorov-Smirnova y Shapiro-Wilk, dando significancias de $\mathrm{p}=0,002$ y $\mathrm{p}=0,009$, respectivamente, por lo cual, se determinó un análisis de datos bajo variables paramétricas, ya que presentan una distribución normal de los datos recolectados.

Al mismo tiempo, el análisis de las varianzas con base en la prueba de Levene's estableció la igualdad de estas a partir los datos obtenidos, comprobándose de este modo la homogeneidad de las varianzas dentro del presente estudio tanto para la PAS como para la PAD, observándose una $\mathrm{p}<0,05$.

Con base en el comportamiento normal se procede al análisis inferencial de los datos, el cual se realizó mediante el programa estadístico para las ciencias sociales SPSS versión 22.0, el cual consistió en evaluar el efecto cardioprotector 
mediante la hipotensión post-ejercicio a dos intensidades y una sesión control, utilizando un diseño de experimentos de 2 vías.

Para las PAS se evidenciaron diferencias significativas en intensidad y tiempo $(\mathrm{p}=0,024$ y $\mathrm{p}=0,016)$, no obstante, la interacción intensidad por tiempo $(\mathrm{p}=0,859)$ no obtuvo estas diferencias. Por otra parte, el análisis de la $\mathrm{PAD}$ no muestra diferencias significativas para estos dos factores, razón por la cual se muestra el efecto hipotensor del ejercicio, principalmente en la PAs en comparación con la PAD.

Por último, mediante la comparación múltiple de los datos se identificó qué tratamientos fueron estadísticamente diferentes, de acuerdo a sus medias, mediante el test de Bonferroni, determinando así diferencias significativas principalmente en el momento inmediatamente posterior a terminado el ejercicio (de 20 a 30 minutos después del mismo) únicamente para la PAs.

\section{Discusión}

Se establece, a partir de los resultados, que el ejercicio aeróbico ejecutado a las dos intensidades estipuladas conlleva un efecto hipotensivo en el individuo, sin embargo, se evidenciaron disminuciones significativas principalmente en la PAs a partir de la estadística inferencial, lo que coincide con los hallazgos de Gamboa y Solera (2014), quienes realizaron el análisis con ejercicio aeróbico a 10 individuos físicamente activos en el que midieron las mismas variables, observando una disminución significativa únicamente en la PAs. No obstante, Álvarez et al., (2013), en su estudio con diferentes grupos etarios, obtuvieron diferencias significativas para la PAS y la PAD en adolescentes y adultos sanos, lo que pudo depender del tiempo de ejercicio (60 min) o de su intensidad — la cual no es estipulada en la publicación — al ser de baja intensidad bajo un método fraccionado. Por el contrario, en el presente estudio no se encontraron diferencias significativas para la PAD.

Por otra parte, la respuesta hipotensiva entre hombres y mujeres no determinó diferencias considerables, aunque se evidencia que las tensiones arteriales de los hombres en el transcurso de los 60 minutos se mantuvieron más cerca a 
los valores de reposo, siendo esto causado, probablemente, por la recuperación más rápida de la PA de los hombres con respecto a las mujeres (Chiacchio, Ricart \& Suau, 2010).

Los participantes del estudio realizaron ejercicio aeróbico en bicicletas estáticas, con un volumen de 30 minutos y una intensidad de 50\% y $70 \%$ de la FC reserva, obteniendo efectos hipotensivos en los dos casos. Lo que confirma lo expuesto por Gomes y Doederlein (2011), quienes mencionan que la relación de actividades aeróbicas, con duraciones entre 30 y 45 minutos, es idónea para provocar HPE. Asimismo, los resultados también coinciden con lo encontrado por Arsa et al. (2006), quienes realizaron un estudio para determinar el efecto hipotensor del ejercicio con intensidad constante frente a la variable del $60 \%$ FC reserva y la otra $50 \%$ y $80 \%$ FC reserva respectivamente, con lo cual, no evidenciaron diferencias significativas en los métodos de ejercicio ejecutados en ese rango de intensidades para obtener una HPE.

Con base en los resultados descriptivos, se evidencia la mayor hipotensión arterial sistólica con intensidad del $70 \%$ en los minutos inmediatamente posteriores al ejercicio (minuto 10), lo que puede ser a causa de la regulación de procesos fisiológicos para que el cuerpo recupere la homeostasis, entre los cuales se puede atribuir a la disminución de las resistencias vasculares periféricas, con lo cual, se produce un descenso de la PA más pronunciado que en la intensidad del $50 \%$, pero sin diferencia significativa entre las dos, aun así, esta disminución no necesariamente pudo ser el mecanismo de hipotensión obtenido en el estudio.

Sin embargo, dentro del efecto cardioprotector del ejercicio aeróbico se establecen otros mecanismos por los cuales pudo existir el efecto hipotensivo, tales como disminución de catecolaminas circundantes (MacDonald, 2002), el aumento de sustancias vasodilatadoras — como lo es el óxido nítrico (Pescatello et al., 2004)_, disminución de la resistencia a la insulina inhibiendo la activación del sistema simpático (Cornelissen \& Fagard, 2005), vasodilatación cutánea (MacDonald, 2002), entre otros; determinando así procesos simultáneos y explicando descriptivamente por qué la PAS y la PAD no regresaron a sus valores iniciales después de 60 minutos posteriores al ejercicio, pero sí evidenciando una recuperación progresiva hacia estos valores de reposo. 
El efecto de protección cardiaca mediante la HPE sumado a los mecanismos anteriormente descritos, y a través de la realización sistemática del ejercicio, pueden ser las adaptaciones estructurales que expliquen a nivel cardiovascular la disminución de la PA en reposo (Pescatello et al., 2004), con lo que se evidencia a través de los resultados que, paralelamente el ejercicio aeróbico, funciona como elemento preventivo para disminuir la incidencia de una futura HTA, concordando con la tesis expuesta por Simão et al. (2007) quienes sustentan la misma afirmación.

Se ha realizado una gran cantidad de estudios con referencia a los efectos hipotensivos, especialmente después del ejercicio aeróbico, en poblaciones normotensas aparentemente sanas e hipertensas. Sin embargo, las poblaciones con factores de riesgo, en este caso de HTA, también presentan HPE cuando son a volúmenes e intensidades adecuadas, lo que ratifica lo expuesto por Carpio $e t$ al. (2015) quienes, mediante una revisión sistemática, evidenciaron una disminución de la PAS y la PAD independientemente del tipo de población de estudio.

\section{Conclusiones}

Se comprueba la incidencia del ejercicio aeróbico sobre la PA en las dos intensidades (50\% y 70\%) mediante la HPE en personas con riesgo de HTA, sin embargo, se determinaron diferencias significativas únicamente en la PAs. Por otra parte, la mayor disminución de la PA posterior al ejercicio se evidenció con la intensidad del 70\% de la FCR, observándose principalmente un efecto agudo (minutos 20 y 30) después de finalizado el ejercicio. No obstante, no se demuestran cambios significativos en la disminución de la PAS entre intensidades del 50 y $70 \%$ FCR.

En cuanto a la HPE, no se evidenciaron diferencias importantes entre hombres y mujeres, sin embargo, llama la atención la respuesta de la PA en la sesión de control, por lo cual, se sugiere la realización de estudios donde se realice esta comparación por géneros con poblaciones más grandes.

Por último, la PA no se recuperó en su totalidad 60 minutos después a la finalización del ejercicio, por ello se recomienda a futuras investigaciones medir la HPE por un periodo de tiempo más prolongado. 


\section{Referencias}

Álvarez, C., Olivo, J., Robinson, O., Quintero, J., Carrasco, V., Ramírez, R., Andrade, D., \& Martínez, C. (2013). Efectos de una sesión de ejercicio aeróbico en la presión arterial de niños, adolescentes y adultos sanos. Revista Médica de Chile, 141(11), 1363-1370.

American College of Cardiology \& American Heart Association (2002). Guideline Update for Exercise Testing. http://www.cardiology.org/recentpapers/exercisegl02.pdf

Arsa, G., Cristina, A., Rodrigues, J., Braga, P., Grubert, C., Simoes, H., \& Ruiz, M. (2006). Post-exercise hypotension in hypertensive individuals submitted to aerobic exercises of alternated intensities and constant intensity-exercise. Revista brasileira de medicina do esporte, 12(6), 281-284.

Briones, E. (2016). Ejercicios físicos en la prevención de hipertensión arterial. Medisan, 29(1), $35-41$.

Calderón, F. (2009). Fisiología aplicada al deporte. Editorial Tébar.

Carpio, E., Moncada, J., Salazar, W., \& Solera, A. (2016). Acute effects of exercise on blood pres\& sure: a meta-analytic investigation. Arquivos Brasileiros de Cardiologia, 106(5), 422-433.

Chiacchio, M., Ricart, A., \& Suau, R. (2010). Blood pressure response to exercise testing. Apunts medicina de l'esport, 45(167), 191-200. https://pdfs.semanticscholar.org/caa6/ 23df59779e2966e04d2b6258dab80f738cd2.pdf

Cornelissen, V., \& Fagard, R. (2005). Effects of endurance training on blood pressure, blood pressure-regulating mechanisms, and cardiovascula risk factors. Hypertension, 46(4), 667-675.

Cornelissen, V., Verheyden, B., Aubert, A., \& Fagard, R. (2010). Guideline Update for Exercise Testing: on resting, exercise and post-exercise blood pressure, heart rate and heart-rate variability. Journal of Human Hypertension, 175-182.

Del Valle, M., Manonelles, P., Galván, C., Franco, L., Luengo, E., \& Gaztańaga, T. (2015). Prescripción de ejercicio físico en la prevención y tratamiento de la hipertensión arterial. Documento de Consenso de la Sociedad Española de Medicina del Deporte (SEMEDFEMEDE). Archivos de medicina del deporte, 32(5), 281-312.

Estañol, B., Gutiérrez, F., Martínez, R., Sentíes, H., Berenguer, M., Magaña, L., Delgado, G., \& Chiquete, E. (2016). Las dos caras del reflejo venoarteriolar: vasoconstricción y vasodilatación cutánea al bajar y subir el brazo. Revista de neurología, 62(9), 403-407.

Gamboa, M., \& Solera, A. (2014). Efecto agudo de dos intensidades de ejercicio aeróbico sobre la presión arterial en reposo de personas normotensas. Revista andaluza de medicina del deporte, 7(3), 101-105.

Gomes, P., \& Doederlein, M. (2011). Hipotensión post-ejercicio en individuos hipertensos: una revisión. Arquivos Brasileiros de Cardiologia, 96(5), 100-109.

González, R., Llapur, R., Díaz, M., Illa, M., Yee, E., \& Pérez, D. (2015). Estilos de vida, hipertensión arterial y obesidad en adolescentes. Revista cubana de pediatría, 87(3), 273-284.

Hernán, A., Marit, S., Oivind, S., Asgeir, M., Lars, L., Elin, E., Reidun, O., \& Bente, U. (2015). Efectos favorables de la actividad física regular motivada en el trabajo sobre la tensión arterial y el perfil lipídico. Medicina y seguridad del trabajo, 61(239), 162-171. 
MacDonald JR, (2002). Potential causes, mechanisms, and implications of post exercise hypotension. Journal of Human Hypertension, 16, 225-236.

Marins, J., \& Delgado, M. (2007). Empleo de ecuaciones para predecir la frecuencia cardiaca máxima en carrera para jóvenes deportistas. Archivos de Medicina del Deporte, 24(118), 112-120.

Márquez, S., \& Garatachea, N. (2009). Actividad física y salud. Ediciones Díaz de Santos.

Ministerio de Salud y Protección Social (2014). Análisis de situación de salud. Colombia 2013. Imprenta Nacional de Colombia.

Ministerio de Salud (1993, 04 de octubre). Resolución 8430. Por la cual se establecen las normas científicas, técnicas y administrativas para la investigación en salud. https:// www.minsalud.gov.co/sites/rid/Lists/BibliotecaDigital/RIDE/DE/DIJ/RESOLUCION8430-DE-1993.PDF

Mora, R. (2010). Fisiología del deporte y el ejercicio. Panamericana.

Pescatello, L., Frankil, B., Fagard, R., Farquhar, W., Kelley, G., \& Ray, C. (ACSM) (2004). Exercise and hypertension. Medicina y ciencia en deportes y ejercicio, 36(3), 533-553.

Simão, R., Deus, J., Miranda, F., Lemos, A., Baptista, L., \& Novaes, J. (2007). Hypotensive effects in hypertenses after judo class. Fitness \& Performance, 6(2), 116-120.

Tanaka, H., Monahan, K., \& Seals, D. (2001). Age-predicted maximal heart rate revisited. Journal of the American College of Cardiology, 37(1), 153-156.

Veloso, J., Polito, M., Riera, T., Celes, R., Vidal, J., \& Bottaro, M. (2010). Efectos del intervalo de recuperación entre las series sobre la presión arterial luego de ejercicios de resistencia. Arquivos Brasileiros de Cardiologia, 94(4), 496-502. 